\title{
Pretreatment with $\omega-3$ fatty acid infusion to prevent leukocyte-endothelial injury responses seen in cardiac surgery
}

Jonathan McGuinness, MD, PhD, John Byrne, MB, BCH, BAO, MRCSI, Claire Condron, PhD, Jim McCarthy, MCh, FRCSI, David Bouchier-Hayes, MCh, FRCSI, and J. Mark Redmond, MD

From the Department of Surgical Research, The Royal College of Surgeons in Ireland, Dublin, Ireland.

Supported by a research fellowship in surgery grant awarded by the Royal College of Surgeons in Ireland.

Received for publication June 2, 2007; revisions received Oct 16, 2007; accepted for publication Nov 13, 2007.

Address for reprints: J. Mark Redmond, MD, The Royal College of Surgeons in Ireland, Department of Surgery, RCSI Education and Research Centre, Beaumont Hospital, Dublin, Ireland (E-mail: mredmond@beaconmedicalgroup.ie).

J Thorac Cardiovasc Surg 2008;136:135-41 $0022-5223 / \$ 34.00$

Copyright (C) 2008 by The American Association for Thoracic Surgery

doi:10.1016/j.jtcvs.2007.11.010
Objective: Inappropriate multiorgan endothelial-leukocyte activation is major causative factor in organ dysfunction after cardiac surgery. We investigated in vitro, mechanism and magnitude of attenuation of the pathogenic response through pretreatment with an $\omega-3$ fatty acid infusion.

Methods: Perioperative saphenous endothelial cell monolayers were pretreated and then stimulated with perioperative inflammatory mediators. Endothelial production of interleukin 6 , interleukin 8 , and adhesion molecules necessary for neutrophil tissue penetration, were examined, together with inflammatory endothelial coagulant responses. Pretreatment effects on isolated blood neutrophil inflammatory responses were similarly noted. Mechanistic insight was obtained through assessment of the temporal response of nuclear factor- $\mathrm{kB}$ and its association with heat shock protein 72(HSP72) expression.

Results: Four-hour pretreatment markedly reduced inflammatory endothelial release of interleukin $8(2587 \pm 82 \mathrm{pg} / \mathrm{mL}$ control vs $208 \pm 3 \mathrm{pg} / \mathrm{mL} \omega-3$ pretreated, $P<$ $.01)$ and endothelial expression of intercellular adhesion molecule $1(196.1 \pm 2.0$ vs $71.9 \pm 0.6$ mean channel fluorescence, $P<.01)$ in response to endotoxin and tumor necrosis factor a. Neutrophil activation (CD11b and respiratory burst) was maintained, but pretreated neutrophils had shorter survival. Endothelial inflammatory stimulation produced rapid increase in nuclear activity of nuclear factor-kB, which was attenuated by $43 \%$ with $\omega-3$ pretreatment $(P<.01)$. This coincided with 3 -fold increase $(P=.03)$ in protective HSP72 expression with pretreatment.

Conclusion: Acute pre-treatment with a clinically acceptable $\omega$-3 infusion attenuates perioperative endothelial-neutrophil activation through transcription-level interaction.

$\mathrm{T}$ he systemic inflammatory response and ischemia-reperfusion have at their core the common pathogenesis of tissue injury caused by excessive leukocyte-endothelial inflammatory activation and interaction. ${ }^{1-5}$ Cardiac surgery generates a prominent systemic inflammatory respons $\mathrm{e}^{2,4-7}$ and necessitates a period of cardiac and pulmonary ischemia-reperfusion injury. Pediatric cardiac surgery is further complicated by the need in many cases for a period of circulatory arrest or low-flow cardiopulmonary bypass in which multiorgan ischemia-reperfusion injury occurs. Leukocyte-endothelial interaction is an evolutionary response that minimizes injury by containing it locally, with some inadvertent normal tissue damage, to prevent systemic complications. In cardiac surgery, because of the systemic activation of the endothelium in multiple organ capillary beds, inadvertent and inappropriate sequestration of leukocytes occurs in multiple organs, producing multiple organ dysfunction syndrome. . $^{2,3,5,8,11}$

The four main components of the endothelial response to inflammatory activation are as follows: production of proinflammatory cytokines to recruit neutrophils and amplify the inflammatory response, upregulation of cell adhesion molecules to allow 


$$
\begin{aligned}
& \text { Abbreviations and Acronyms } \\
& \begin{aligned}
\text { HSP } & =\text { heat shock protein } \\
\text { ICAM-1 } & =\text { intercellular adhesion molecule } 1 \\
\text { IL } & =\text { interleukin } \\
\text { LPS } & =\text { lipopolysaccharide } \\
\text { mcf } & =\text { mean channel fluorescence } \\
\text { NF- } \kappa \mathrm{B} & =\text { nuclear factor } \kappa \mathrm{B} \\
\mathrm{TNF}-\alpha & =\text { tumor necrosis factor } \alpha
\end{aligned}
\end{aligned}
$$

leukocyte adherence and transmigration, generation of a procoagulant environment at the endothelial level, and vasomotor dysfunction. ${ }^{2,3,7}$ To date, most potential strategies targeted against the central process of leukocyte-endothelial activation have focused on inhibiting a single aspect of this response, such as oligosaccharide antagonists or monoclonal antibodies against the adhesion molecules on the endothelium or leukocytes, ${ }^{3,10,12,13}$ antioxidants to counter neutrophil attack, ${ }^{5}$ steroids to prevent neutrophil activation, ${ }^{5}$ or leukofiltration. ${ }^{3,5}$

Pure forms of $\omega-3$ fatty acids have been shown to inhibit inflammation-induced expression of leukocyte adhesion molecules on the endothelium. ${ }^{14,15}$ This study examined whether a clinically acceptable form of $\omega-3$ fatty acids that could be used prophylactically in the setting of cardiac surgery might produce a similar effect in vitro on cell adhesion molecule expression, as well as the effect on neutrophilrecruiting cytokines, endothelial procoagulant response, and neutrophil inflammatory responses, and whether this effect would arise through a preconditioning type of mechanism.

\section{Materials and Methods}

Ethical approval for the study was granted by the Mater Misericordiae University Hospital, with written informed consent obtained from 75 patients undergoing coronary bypass surgery to use any remnant saphenous vein for endothelial cell isolation. ${ }^{9}$ Young healthy volunteers consented to donate a peripheral blood sample for neutrophil isolation.

\section{Endothelial Cell and Neutrophil Isolation}

Endothelial cells were isolated from the remnant saphenous vein as described previously. ${ }^{16}$ Medium containing endothelial cell growth factor (neural bovine extract endothelial cell growth factor; Sigma-Aldrich, St. Louis, MO, USA) at a concentration of $8 \mathrm{mg} / 100$ $\mathrm{mL}$ was added. Cells were grown to the fourth passage for experiments, and endothelial purity was confirmed by greater than $95 \%$ staining with von Willebrand factor (Dako North America, Inc., Carpinteria, CA, USA). The cells used in experiments therefore had not been exposed to any medicinal products or to high blood glucose levels (as in patients with diabetes), minimizing potential confounding variables.

Neutrophils were isolated from peripheral blood samples immediately by dextran (Sigma-Aldrich) density sedimentation and Ficoll (Amersham plc, Little Chalfont, UK) gradient centrifugation, as described previously. ${ }^{17}$ The neutrophils were suspended in complete medium: Roswell Park Memorial Institute medium containing $10 \%$ volume/volume fetal calf serum, $1 \%$ volume/volume penicillin-streptomycin solution (GIBCO; Life Technologies Ltd, Paisley, UK), and placed as aliquots into polypropylene tubes (Sarstedt, Numbrecht, Germany). These techniques minimize activation of neutrophils as assessed by size versus granularity dot plots and yield neutrophil purity greater than $95 \%$ according to CD15/CD16 staining on flow cytometry.

\section{Model of Systemic Inflammatory Activation}

Endothelial cells were transferred to 12-well plates for experiments, with $1 \times 10^{5}$ cells/well in a confluent monolayer. Neutrophils were used immediately suspended at $2 \times 10^{6}$ cells $/ \mathrm{mL}$ medium in polypropylene tubes (Starstedt). Normal medium or medium containing a 1:400 dilution of the parenteral nutrition formulation of $\omega-3$ infusion (Omegaven; Fresenius Kabi AG, Bad Homburg, Germany) was added to each well or tube for 4 hours, the minimum period of required exposure noted in previous experiments with isolated macrophages. ${ }^{18}$ After 4 hours, the medium was removed and 1 $\mathrm{mL}$ medium containing lipopolysaccharide (LPS) at $1 \mu \mathrm{g} / \mathrm{mL}$ (Sigma-Aldrich), tumor necrosis factor $\alpha$ (TNF- $\alpha$ ) at $0.03 \mu \mathrm{g} / \mathrm{mL}$ (RnD, UK), or complement component C5a at $0.1 \mu \mathrm{g} / \mathrm{mL}$ (EMD Chemicals, Inc., San Diego, CA, USA) was added.

\section{Cell Adhesion Molecule Expression}

Flow cytometry was used to measure changes in adhesion molecule expression, with appropriate isotypic controls. Endothelial E-selectin (CD62E) and intercellular adhesion molecule 1 (ICAM-1, CD54), and neutrophil CD11b/CD18 were assessed with mouse antihuman R-Phycoerythrin-conjugated monoclonal antibodies (BD Bioscience, UK). Receptor density on the cell surface was expressed as mean channel fluorescence (mcf) intensity of the cells.

\section{Endothelial Coagulant Changes}

Endothelial surface thrombomodulin levels were assessed with flow cytometry with a primary mouse antihuman thrombomodulin monoclonal antibody (CD141; American Diagnostica Inc, Greenwich, Conn), and a secondary fluorescein isothiocyanate-conjugated sheep antimouse IgG (Bioquote Limited, North Yorkshire, UK). Supernatant thrombomodulin and tissue factor levels were measured with the Imubind thrombomodulin and tissue factor enzyme-linked immunosorbent assay kits (American Diagnostica).

\section{Proinflammatory Cytokine Release}

Supernatants from the endothelial and neutrophil experiments were used to measure release of the proinflammatory cytokines interleukin (IL) 6, IL-8, and TNF- $\alpha$ with enzyme-linked immunosorbent assay kits $(\mathrm{RnD})$.

\section{Neutrophil Respiratory Burst}

Intracellular reactive oxygen intermediate generation by neutrophils was assessed with the Orpegen BurstTest Kit (BD Becton Dickinson UK Limited, Oxford, UK). Briefly, neutrophils stimulated with phorbol 12-myristate 13-acetate were exposed to dihydrorhodamine 123 to label reactive species for visualization with flow cytometry, and intensity was expressed as mcf. 


\section{Endothelial Cell and Neutrophil Apoptosis and Viability}

Flow cytometric measurements of dual staining with annexin $\mathrm{V}$ and propidium iodide were used to assess the percentage of cells that were apoptotic or viable (TACS Apoptosis Detection Kit; RnD). For neutrophils, because of ongoing apoptosis and short lifespan, exposure to LPS at $1 \mu \mathrm{g} / \mathrm{mL}$ (Sigma-Aldrich) and complement component C5a at $0.1 \mu \mathrm{g} / \mathrm{mL}$ (Calbiochem) for 20 hours was necessary to be able to detect the normal prolongation of neutrophil survival that occurs with inflammation and consequently assess the effect of $\omega-3$ pretreatment.

\section{Endothelial Nuclear Factor $\kappa B$ and Heat Shock Protein 72 Expressions}

The relative concentrations of the two active subunits of the acute inflammatory transcription factor nuclear factor $-\kappa \mathrm{B}(\mathrm{NF}-\kappa \mathrm{B})$, ie $\mathrm{P} 65$ and P50, were measured in endothelial cytoplasmic and nuclear proteins at different points with the TransAM NF- $\kappa$ B Chemi Kit (Active Motif, Rixensart, Belgium). Cytoplasmic and nuclear proteins were isolated with a nuclear extract kit (Active Motif), protein concentrations were equalized with the Bradford assay (SigmaAldrich), and samples were placed in wells coated with an oligonucleotide containing the NF- $\kappa \mathrm{B}$ consensus binding site that would be present in the $5^{\prime}$ promoter region of the inflammatory genes activated by $\mathrm{NF}-\kappa \mathrm{B}$. A sandwich enzyme-linked immunosorbent assay technique was then used to detect p65 or p50 binding to this site. Results are expressed as relative luminescence units. Heat shock protein (HSP) 72 levels in the cytoplasmic protein extracts were assessed with Western blotting with samples at the same points as the $\mathrm{NF}-\kappa \mathrm{B}$ experiments for comparison of results. The primary antibody was a mouse antihuman HSP72 monoclonal antibody (SPA-810; Bioquote), and the secondary was a horseradish peroxidase-conjugated goat antimouse $\operatorname{IgG}_{1}$ (DAKO). The relative densitometric readings for each blot were normalized with $\beta$-actin (antihuman beta actin antibody; Oncogene Research Products, Merck).

\section{Statistical Analysis}

Results are expressed as mean \pm SEM. Data were analyzed with a 1-way analysis of variance, with post hoc comparisons performed with the Bonferroni comparison of means as appropriate.

\section{Results}

\section{Endothelial Effects of $\omega-3$ Pretreatment}

Pretreatment for 4 hours with the $\omega-3$ infusion markedly attenuated release of the potent neutrophil-recruiting cytokine IL-8 and the proinflammatory cytokine IL- 6 after 3 hours of inflammatory stimulation with LPS or TNF$\alpha$ (Figure 1). Pretreatment also prevented upregulation of molecules for neutrophil adhesion on the endothelial surface, both E-selectin for neutrophil rolling on the endothelium and ICAM-1 for firm neutrophil adherence and degranulation (Figure 2). Although absolute levels of adhesion molecules were not measured, but rather changes in mcf were used, the lack of any change in mcf with $\omega-3$ pretreatment indicates that the endothelium behaves as if it were unstimulated by TNF or LPS. The endothelial procoagulant response to inflammation is normally characterized by a reduction in the endothelial surface anticoagulant thrombomodulin and an increase in procoagulant tissue factor release by the endothelium. ${ }^{2,7}$ Endothelial surface thrombomodulin levels were noted to be reduced with inflammatory stimulation with LPS $(57.3 \pm 0.7 \mathrm{mcf}$ control vs $49.9 \pm 0.3 \mathrm{mcf}$ LPS stimulation) but were maintained at baseline levels if the endothelium was pretreated with $\omega-3$ fatty acids $(64.9 \pm 4.9 \mathrm{mcf}, P<$ .01). Supernatant thrombomodulin levels at the same point showed no changes in thrombomodulin levels with LPS stimulation $(0.202 \pm 0.004 \mathrm{ng} / \mathrm{mL}$ vs baseline $0.207 \pm 0.007 \mathrm{ng} /$ $\mathrm{mL}, P=.8$ ), so the observed reduction in thrombomodulin levels on the inflammation-activated endothelial surface likely represents reduced expression, rather than shedding of the thrombomodulin. Supernatant tissue factor levels were undetectable.

\section{Endothelial Viability and Reversibility}

It was important to confirm that the reduction in endothelial inflammatory response that occurred with $\omega-3$ pretreatment was not due simply to a reduction in endothelial viability. Pretreatment with a 1:400 dilution of the $\omega-3$ infusion for 4 hours had no significant effect on endothelial viability relative to untreated cells (Table 1). A time course experiment with ICAM-1 upregulation in response to LPS stimulation as the end point was used to assess whether the antiinflammatory effects of $\omega-3$ pretreatment were reversible. Endothelial monolayers were pretreated with the $\omega-3$ infusion for 4 hours and then exposed to LPS either immediately or at a delayed interval, and ICAM-1 was measured. Endothelial responsiveness to LPS stimulation returned between 18 to 24 hours after pretreatment (Figure 3).

\section{Effects of $\omega-3$ pretreatment on Neutrophil Acute Inflammatory Responses}

Pretreatment with the $\omega-3$ infusion did not attenuate the neutrophil's ability to generate reactive oxygen species, express the endothelial adhesion molecule CD11b/CD18, or release IL-8 in response to inflammatory stimulation. Neutrophils have a half-life in the circulation of 6 hours because of an ongoing process of apoptosis; this ensures that any potential damaging response is short-lived. ${ }^{19}$ During cardiac surgery, this normal rate of apoptosis is reduced, increasing the neutrophil's lifespan not only in the circulation but also potentially within the tissues and thus prolonging the period of inadvertent tissue damage. ${ }^{19}$ Stimulation of isolated neutrophils with LPS and C5a for 20 hours increased neutrophil survival as a result of reduced apoptosis, as expected. Pretreatment with a 1:400 dilution of the $\omega-3$ infusion for 4 hours maintained nearly normal neutrophil survival (Figure 4).

\section{Mechanism of Endothelial Anti-inflammatory Effects} Changes in both the p50 and p65 active subunits of the acute inflammatory transcription factor NF- $\kappa$ B showed the same 

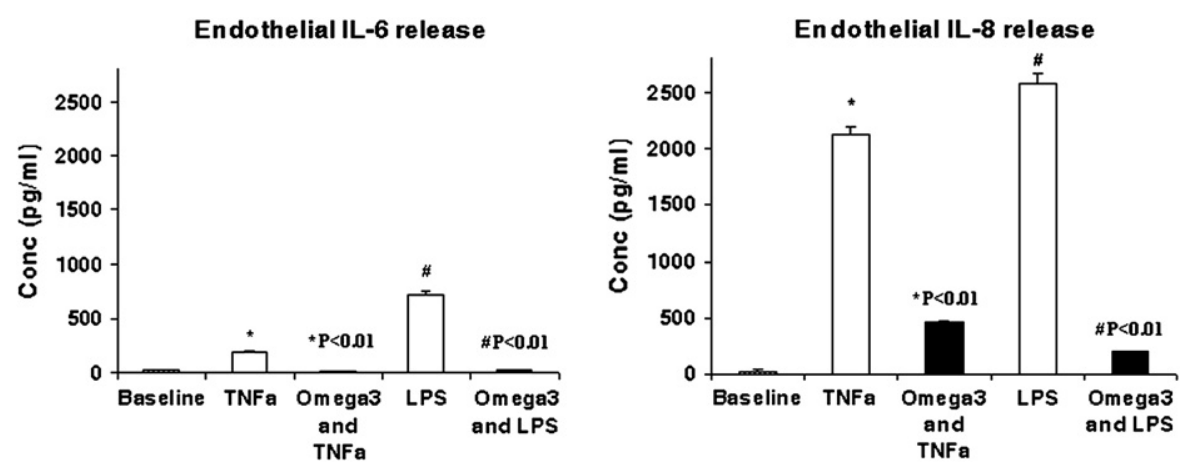

Figure 1. Endothelial cells were stimulated with tumor necrosis factor $\alpha$ (TNFa) or endotoxin (LPS) for 3 hours, and supernatant interleukin 6 (IL-6) and 8 (IL-8) levels were measured. Pretreatment (Omega3) markedly suppressed interleukin 6 and 8 release relative to no pretreatment $(\boldsymbol{P}<.01$ in all cases $)$. pattern, with a 13-fold increase in nuclear levels of the p65 subunit of NF- $\kappa \mathrm{B}$ on LPS stimulation that was markedly attenuated with $\omega-3$ pretreatment to $57 \%$ of the control LPS-stimulated levels $(P<.01$; Figure 5$)$. Endothelial cytoplasmic HSP72 levels were assessed at the same points used in the $\mathrm{NF}-\kappa \mathrm{B}$ assessments. After 4 hours of pretreatment, a significant 3-fold increase in HSP72 levels was noted in the $\omega-3$-pretreated group $(307 \% \pm 60 \%$ vs control $100 \%$, $P=.03)$. After 30 minutes of exposure to LPS, there was little detectable HSP72 in the $\omega-3$-pretreated group but 7-fold
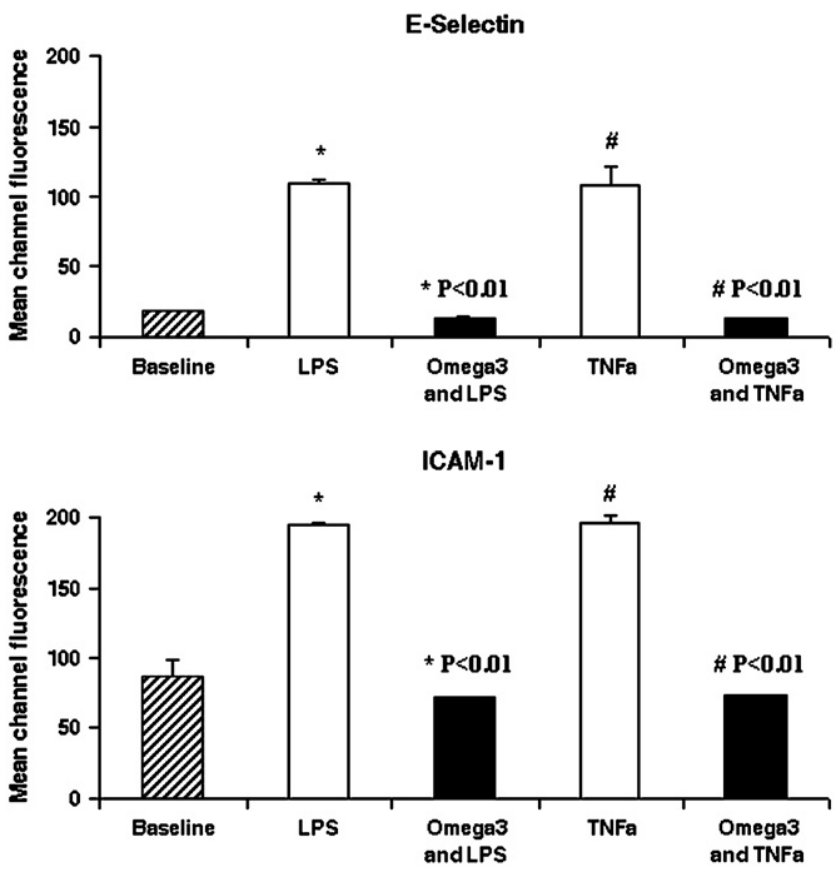

Figure 2. Endothelial cells were stimulated with tumor necrosis factor $\alpha$ (TNFa) or endotoxin (LPS) for 3 hours, and cell surface levels of E-selectin (for neutrophil rolling on endothelium) and intercellular adhesion molecule 1 (ICAM-1, for neutrophil firm adhesion on the endothelium) were measured. Pretreatment (Omega3) prevented upregulation of neutrophil adhesion molecules on endothelium ( $P<.01$ for all comparisons). higher levels in the control group when both were expressed as a percentage of the control 4-hour pretreatment levels $(31 \% \pm 19 \%$ in the $\omega-3-$ pretreated group vs $244 \% \pm 75 \%$ in the control group, $P=.05$; Figure 6).

\section{Discussion}

The link between organ dysfunction and leukosequestration in cardiac surgery is well established. This study illustrates that pretreatment with a clinically acceptable $\omega-3$ infusion can attenuate endothelial release of the neutrophil-recruiting cytokine IL-8 and expression of neutrophil adhesion molecules on the endothelial surface, which may minimize inappropriate perioperative leukocyte penetration of organs. This $\omega-3$ pretreatment also attempts to preserve the normal endothelial anticoagulant state through prevention of inflammation-induced reduction in endothelial surface anticoagulant thrombomodulin expression, which may improve postoperative organ perfusion. Pretreatment does not interfere with important neutrophil bactericidal functions of reactive oxygen species generation and CD11b/CD18 expression. Pretreatment effects on neutrophil adhesion molecule expression by the endothelium appear to be reversible within 24 hours. These pretreatment effects appear to be mediated at transcription level through attenuated activation of the acute inflammatory transcription factor NF- $\kappa$ B. Effects on $\mathrm{NF}-\kappa \mathrm{B}$ activation have a temporal correlation with HSP72 expression, suggesting a preconditioning type of attenuation of inflammatory $\mathrm{NF}-\kappa \mathrm{B}$ activation.

TABLE 1. Endothelial cell populations with and without $\omega-3$ fatty pretreatment

\begin{tabular}{lrcc}
\hline & \multicolumn{1}{c}{ Control } & $\boldsymbol{\omega}$-3 Pretreatment & $\boldsymbol{P}$ value \\
\hline Viable & $87 \% \pm 1 \%$ & $80 \% \pm 1 \%$ & .5 \\
Early apoptotic & $8 \% \pm 1 \%$ & $11 \% \pm 1 \%$ & .8 \\
Necrotic or late apoptotic & $5 \% \pm 1 \%$ & $9 \% \pm 3 \%$ & .6 \\
\hline
\end{tabular}

Endothelial cells were exposed to medium containing a 1:400 dilution of the $\omega-3$ infusion or medium only for 4 hours. Percentages of cells that were viable, were in early apoptosis, or were necrotic or in late apoptosis were assessed. 


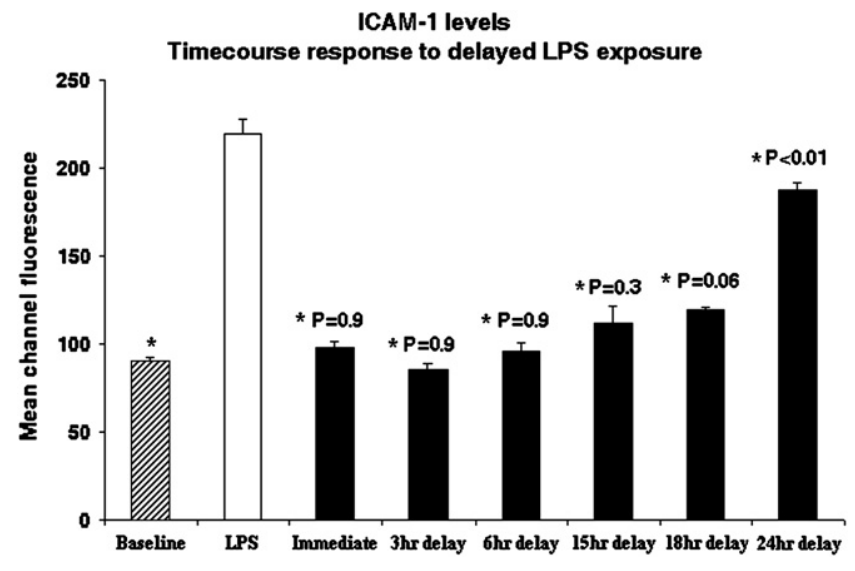

Figure 3. Endothelial cells were exposed to medium only or medium containing 1:400 dilution of u-3 infusion for 4 hours. Subsequently, they were stimulated with endotoxin (LPS) immediately or after a period of exposure to medium only (delayed exposure), and intercellular adhesion molecule 1 (ICAM-1) levels were measured. Normal inflammatory upregulation of intercellular adhesion molecule 1 returned, 18 to 24 hours after $\omega-3$ pretreatment. All levels are compared to baseline unstimulated endothelial cells $(*)$.

Neutrophils possess some of the most toxic proteases and reactive oxygen species in the body, making them efficient at destroying invading pathogens and damaged nonfunctional tissue. $^{20}$ They lack, however, the ability to differentiate between foreign and host antigens, depending on the localized upregulation of adhesion molecules at a site of infection or

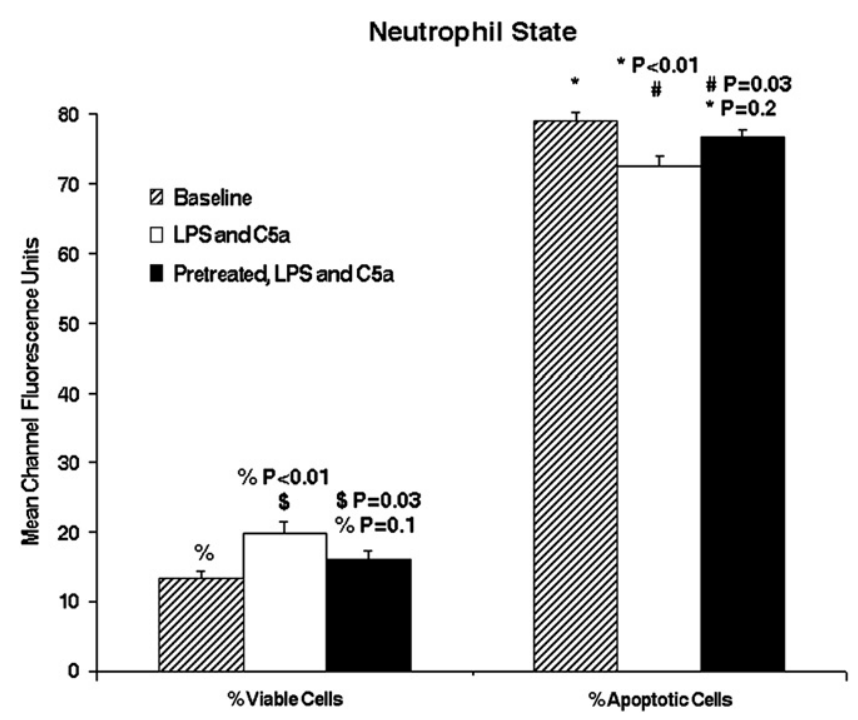

Figure 4. Neutrophils were stimulated with endotoxin (LPS) and complement C5a for 20 hours and assessment of viability or apoptosis. Pretreatment prevented inflammation-induced increase in viability through maintenance of baseline apoptosis levels.

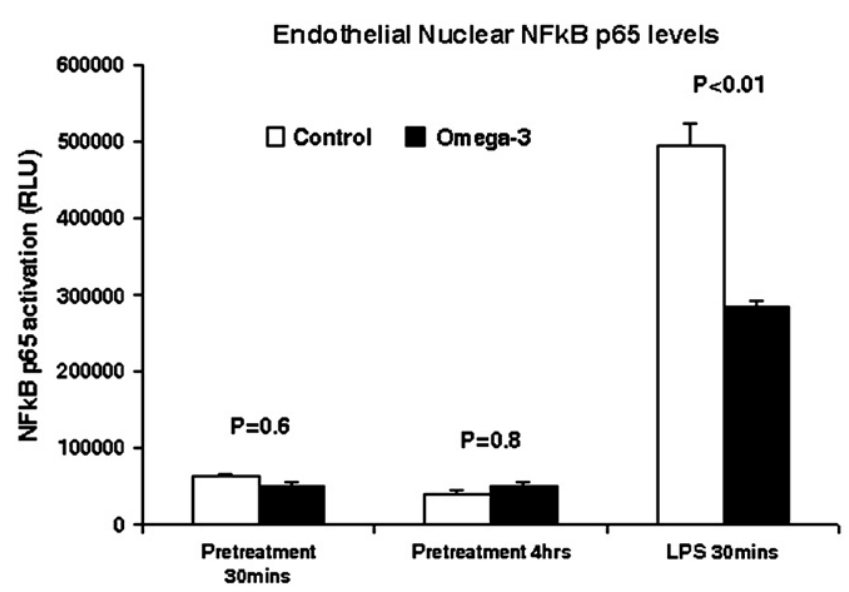

Figure 5. Relative levels of p65 subunit of nuclear factor $\kappa B$ (NFkB) in nucleus after pretreatment of endothelial cells with medium only or medium containing 1:400 dilution of $\omega-3$ infusion (Omega-3) and subsequent inflammatory stimulation with endotoxin (LPS) containing medium for $\mathbf{3 0}$ minutes. Pretreatment attenuated nuclear factor $\kappa B$ activation. $R L U$, Relative luminescence units.

injury to guide them to the appropriate area. Once they have entered the tissues, the release of their stored toxins is not cell specific, and surrounding normal tissue is inadvertently destroyed in the process, although because this is a localized response, collateral injury is minimized. ${ }^{20}$ After cardiac surgery, however, the large number of circulating inflammatory mediators, or multiorgan ischemia-reperfusion in the case of circulatory arrest or low-flow bypass, unfortunately means that multiorgan endothelial activation occurs. $^{2,3,5}$ This converts a localized protective response into

\section{Endothelial Cytoplasmic HSP72 Levels}

\section{Pretreatment}

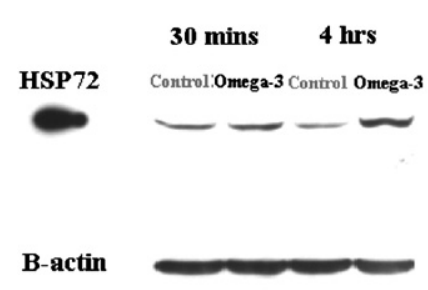

Figure 6. Endothelial cytoplasmic heat shock protein 72 (HSP72) levels were assessed by Western blotting with their associated $\beta$-actin (B-actin) levels. There was significant upregulation of HSP72 after 4 hours of pretreatment with 1:400 dilution of $\omega$-3 infusion (Omega-3). Opposite trend occurred after 30 minutes of exposure to endotoxin (LPS), with higher heat shock protein 72 levels in control group, suggesting more injury and activation of control endothelium. 
a multiorgan pathogenic response, which in the presence of minimal preoperative organ reserve function can lead to organ dysfunction or failure.

Pure forms of $\omega-3$ fatty acids are known to suppress inflammation-induced expression of leukocyte adhesion molecules on the endothelium ${ }^{14,15}$ and IL- 6 and IL-8 production by the activated endothelium, ${ }^{14}$ leading in turn to a reduction in endothelial transmigration of leukocytes as assessed by intravital microscopy. ${ }^{15}$ This study demonstrates that a clinically acceptable parenteral nutrition formulation of $\omega-3$ infusion can replicate these effects with only 4 hours of pretreatment. This protection is reversible, likely lasting only 18 to 24 hours, which reduces the potential for any adverse side effects.

The inflammation-activated endothelium develops into a procoagulant phenotype, presumably to contain any infection, tissue injury, or neutrophil-mediated tissue destruction locally and to minimize the endothelial inflammatory leakage by inducing small areas of microcirculatory thrombosis. ${ }^{21}$ The process is normally localized to a specific site through control mediated by changes in the localized endothelium from a normal anticoagulant surface, provided in part by thrombomodulin expression, to a procoagulant surface, aided by tissue factor production by the endothelium. ${ }^{2,7,23}$ Again, in cardiac surgery, the fact that inflammatory activation of the endothelium occurs in multiple organ capillary beds leads to a normally protective inflammatory response's conversion to a pathologic response, with diffuse downregulation of endothelial surface thrombomodulin and diffuse upregulation of endothelial tissue factor and platelet-activating factor production. ${ }^{22}$ In this study, the normal decrease in cell surface thrombomodulin that occurs with LPS stimulation of the endothelium was prevented by pretreatment with the $\omega-3$ infusion, which appears to downregulate endothelial surface expression. Clearly, $\omega-3$ pretreatment may have a beneficial effect in improving organ perfusion by preventing the noreflow phenomenon both through maintaining endothelial surface anticoagulation in the microcirculation and through inhibiting leukocyte adherence and obstructing accumulation.

This study also illustrates that although pretreatment with the $\omega-3$ infusion has marked anti-inflammatory effects on the endothelium, neutrophil function is largely preserved. Pretreatment with the $\omega-3$ infusion did not affect respiratory burst by the neutrophil, CD11b expression, or proinflammatory cytokine production. Interestingly, the only aspect of neutrophil function that was altered with $\omega-3$ pretreatment was inflammation-induced prolongation of neutrophil survival, which was markedly attenuated as a result of maintenance of near basal levels of apoptosis with pretreatment. This effect would be beneficial in cardiac surgery because it would reduce the survival of postoperatively primed neutrophils while maintaining critical bactericidal functions. Previous studies with oral supplementation with eicosapentaenoic and docosahexaenoic acid for 4 weeks have reported no effect on neutrophil cytokine production or adhesion molecule expression. ${ }^{24}$ These findings are consistent with ours with respect to neutrophil apoptosis, with demonstration in the neutrophil-like differentiated HL-60 cell line that pretreatment with pure $\omega-3$ fatty acids increases apoptosis. ${ }^{25}$

Many of the inflammatory stimuli that activate endothelial cells-including endotoxin (LPS), complement, reactive oxygen species, and proinflammatory cytokines such as IL$1 \mathrm{~b}$, TNF- $\alpha$, and IL- 6 - converge on the transcription factor $\mathrm{NF}-\kappa \mathrm{B}$ within the cytoplasm and activate it, leading to upregulation of the key endothelial inflammatory response genes: genes for adhesion molecules (E-selectin, ICAM-1), genes for procoagulants (tissue factor, plasminogen activator), and genes for proinflammatory factors (TNF- $\alpha$, IL-8, IL-6, IL- 1 , cyclooxygenase 2). ${ }^{22,26}$ This finding underlies the central role for NF- $\kappa \mathrm{B}$ in the systemic inflammatory response and ischemia-reperfusion injury. Because of its critical role, it is important that NF- $\kappa \mathrm{B}$ is maintained in an inactive state within the endothelial cytoplasm, which is achieved by binding of another subunit to the NF- $\kappa \mathrm{B}$ p65-p50 dimer, inhibitory $\kappa \mathrm{B} .{ }^{5,22,26}$

In this study, pretreatment with a 1:400 dilution of the $\omega-3$ infusion was capable of significantly attenuating an LPSstimulated rise in nuclear levels of the active NF- $\kappa$ B subunits, suggesting inhibition at transcription level. Previous studies have shown inhibition of NF- $\kappa$ B by oxidized $\omega-3$ eicosapentaenoic acid ${ }^{15}$ and unoxidized docosahexaenoic acid ${ }^{14}$ within the endothelium. Additionally, pretreatment with this clinically acceptable $\omega-3$ infusion has been shown in isolated murine macrophages to attenuate proinflammatory cytokine production associated with inhibition of NF- $\kappa \mathrm{B} .{ }^{18}$ Our finding of a temporal association between the 3 -fold increase in HSP72 levels in the cytoplasm after 4 hours of pretreatment and the subsequent reduction in NF- $\kappa \mathrm{B}$ activation is interesting. It may suggest a role for HSP72 in preventing NF- $\kappa$ B activation within the cytoplasm. This phenomenon has also been observed in vivo; LPS pretreatment upregulates HSP72 in rats, which produces smaller myocardial infarcts in association with lower levels of NF- $\kappa \mathrm{B}$ activation within the heart. ${ }^{27}$ This may be a direct effect of HSP72 through its molecular chaperoning functions maintaining NF- $\kappa \mathrm{B}$ inactive. ${ }^{28}$ In our study, we also noted that although the $\omega$ 3 infusion produced an increase in cytoplasmic HSP72 relative to control levels, after 30 minutes of LPS stimulation there were actually reduced HSP72 levels in the $\omega-3$ group and 7-fold higher levels in the control group. These findings are easily explained by the fact that HSP70 accumulation can also be used as a biomarker of cellular injury and can reach higher cytoplasmic levels within minutes after physiologic stress. ${ }^{29}$ The temporal alignment of HSP72 expression with $\omega-3$ pretreatment and the subsequent attenuation of NF- $\kappa$ B translocation suggest induction of preconditioning within the endothelium to attenuate its inflammatory activation. The $\omega-3$ infusion likely also acts through other mediators, such as the resolvins and protectins. 
In adult cardiac surgery, there has been a $30 \%$ increase in predicted operative risk during the last decade. ${ }^{30}$ As the age of patients referred for cardiac surgery increases, and the incidence of conditions such as diabetes that produce occult end-organ damage increases in the population in general, the patients now being considered for surgery have more limited physiologic reserves within their organ systems. In pediatric cardiac surgery, newborn children with vulnerable developing organ systems undergo complex procedures with prolonged bypass times and often circulatory arrest or low-flow bypass. Challenging both these patient groups with cardiac surgical systemic inflammatory response and ischemia-reperfusion injury can create dramatic increases in postoperative multiple organ dysfunction or even organ failure. Pretreatment with a clinically acceptable $\omega-3$ infusion for only 4 hours attenuated one of the major pathogenic responses underlying this injury, and this treatment deserves further investigation in a cardiopulmonary bypass and circulatory arrest model as a clinical strategy to improve outcome in our high-risk patient population.

\section{References}

1. Park JL, Lucchesi BR. Mechanisms of myocardial reperfusion injury. Ann Thorac Surg. 1999;68:1905-12.

2. Verrier ED, Boyle EM. Endothelial cell injury in cardiovascular surgery. Ann Thorac Surg. 1996;62:915-22.

3. Boyle EM, Pohlman TH, Johnson MC, Verrier ED. Endothelial cell injury in cardiovascular surgery: the systemic inflammatory response. Ann Thorac Surg. 1997;63:277-84.

4. Elliott MJ, Finn AH. Interaction between neutrophils and endothelium. Ann Thorac Surg. 1993;56:1503-8.

5. Paparella D, Yau TM, Young E. Cardiopulmonary bypass induced inflammation: pathophysiology and treatment. An update. Eur J Cardiothorac Surg. 2002;21:232-44.

6. Asimakopoulos G, Smith PL, Ratnatunga CP, Taylor KM. Lung injury and acute respiratory distress syndrome after cardiopulmonary bypass. Ann Thorac Surg. 1999;68:1107-15.

7. Laffey JG, Boylan JF, Cheng DC. The systemic inflammatory response to cardiac surgery: implications for the anesthesiologist. Anesthesiology. 2002;97:215-52.

8. Sinclair DG, Haslam PL, Quinlan GJ, Pepper JR, Evans TW. The effect of cardiopulmonary bypass on intestinal and pulmonary endothelial permeability. Chest. 1995;108:718-24.

9. Tönz M, Mihaljevic T, von Segesser LK, Fehr J, Schmid ER, Turina MI. Acute lung injury during cardiopulmonary bypass. Are the neutrophils responsible? Chest. 1995; 108:1551-6.

10. Curtis WE, Gillinov AM, Wilson IC, Bator JM, Burch RM, Cameron DE, et al. Inhibition of neutrophil adhesion reduces myocardial infarct size. Ann Thorac Surg. 1993;56:1069-72.

11. Iadecola C. Bone marrow spawns brain killers. Nat Med. 2004;10: 1044-5.

12. Simpson PJ, Todd RF, Mickelson JK, Fantone JC, Gallagher KP, Lee KA, et al. Sustained limitation of myocardial reperfusion injury by a monoclonal antibody that alters leukocyte function. Circulation. 1990;81:226-37.
13. Simpson PJ, Todd RF, Fantone JC, Mickelson JK, Griffin JD, Lucchesi BR. Reduction of experimental canine myocardial reperfusion injury by a monoclonal antibody (anti-Mo1, anti-CD11b) that inhibits leukocyte adhesion. J Clin Invest. 1988;81:624-9.

14. De Caterina R, Liao JK, Libby P. Fatty acid modulation of endothelial activation. Am J Clin Nutr. 2000;71(1 Suppl):213S-23.

15. Sethi S, Ziouzenkova A, Ni H, Wagner DD, Plutzky J, Mayadas TN. Oxidized omega-3 fatty acids in fish oil inhibit leukocyte-endothelial interactions through activation of PPAR $\alpha$. Blood. 2002;100:1340-6.

16. Gosling M, Harley SL, Turner RJ, Carey N, Powell JT. Human saphenous vein endothelial cells express a tetrodotoxin-resistant, voltage-gated sodium current. J Biol Chem. 1998;273:21084-90.

17. Condron C, Toomey D, Casey RG, Shaffii M, Creagh T, BouchierHayes D. Neutrophil bactericidal function is defective in patients with recurrent urinary tract infections. Urol Res. 2003;31:329-34.

18. Babcock TA, Helton WS, Anwar KN, Zhao YY, Espat NJ. Synergistic anti-inflammatory activity of omega-3 lipid and rofecoxib pretreatment on macrophage proinflammatory cytokine production occurs via divergent NF- $\kappa$ B activation. JPEN J Parenter Enteral Nutr. 2004;28:232-9.

19. Power CP, Wang JH, Manning B, Kell MR, Aherne NF, Wu QD, et al. Bacterial lipoprotein delays apoptosis in human neutrophils through inhibition of caspase-3 activity: regulatory roles for CD14 and TLR-2. J Immunol. 2004;173:5229-37.

20. Weiss SJ. Tissue destruction by neutrophils. N Engl J Med. 1989;320: 365-76.

21. Beal AL, Cerra FB. Multiple organ failure syndrome in the 1990s. Systemic inflammatory response and organ dysfunction. JAMA. 1994; 271:226-33

22. Boyle EM, Canty TG, Morgan EN, Yun W, Pohlman TH, Verrier ED. Treating myocardial ischemia-reperfusion injury by targeting endothelial cell transcription. Ann Thorac Surg. 1999;68:1949-53.

23. Esmon NL, Owen WG, Esmon CT. Isolation of a membrane-bound cofactor for thrombin-catalyzed activation of protein C. J Biol Chem. 1982;257:859-64

24. Kew S, Mesa MD, Tricon S, Buckley R, Minihane AM, Yaqoob P. Effects of oils rich in eicosapentaenoic and docosahexaenoic acids on immune cell composition and function in healthy humans. Am J Clin Nutr. 2004;79:674-81.

25. Healy DA, Watson RW, Newsholme P. Polyunsaturated and monounsaturated fatty acids increase neutral lipid accumulation, caspase activation and apoptosis in a neutrophil-like, differentiated HL-60 cell line. Clin Sci (Lond). 2003;104:171-9.

26. Read MA, Whitley MZ, Williams AJ, Collins T, NF-kappa B. I kappa B alpha: an inducible regulatory system in endothelial activation. $J$ Exp Med. 1994;179:503-12.

27. Shimizu M, Tamamori-Adachi M, Arai H, Tabuchi N, Tanaka H, Sunamori M. Lipopolysaccharide pretreatment attenuates myocardial infarct size: a possible mechanism involving heat shock protein 70 inhibitory $\kappa \mathrm{B} \alpha$ complex and attenuation of nuclear factor $\kappa \mathrm{B}$. J Thorac Cardiovasc Surg. 2002;124:933-41.

28. Heneka MT, Sharp A, Klockgether T, Gavrilyuk V, Feinstein DL. The heat shock response inhibits NF- $\kappa$ B activation, nitric oxide synthase type 2 expression, and macrophage/microglial activation in brain. J Cereb Blood Flow Metab. 2000;20:800-11.

29. Benjamin IJ, McMillan DR. Stress (heat shock) proteins: molecular chaperones in cardiovascular biology and disease. Circ Res. 1998;83: $117-32$.

30. Ferguson TB, Hammill BG, Peterson ED, Delong ER, Grover FL. A decade of change-risk profiles and outcomes for isolated coronary artery bypass grafting procedures, 1990-1999: a report from the STS National Database Committee and the Duke Clinical Research Institute. Society of Thoracic Surgeons. Ann Thorac Surg. 2002;73:480-90. 